\title{
Session Delay in File Server Output Buffers with General Session Lengths
}

\author{
Bart Feyaerts, Stijn De Vuyst, Sabine Wittevrongel and Herwig Bruneel \\ SMACS Research Group, TELIN Department, Ghent University \\ Sint-Pietersnieuwstraat 41, 9000 Gent, Belgium \\ E-mail: $\{$ bfeyaert, sdv, sw, hb\}@telin.ugent.be
}

\begin{abstract}
In this paper, we analyze the delay incurred by session-based traffic in the output buffer of a file server.

Users can start and end sessions during which they are active and download information from the file server. Per time slot, each active user downloads a random but strictly positive number of information packets. Each session lasts for a random, yet again, strictly positive number of slots.

We model the file server output buffer as a discrete-time infinite-capacity queueing system and we present an analytical technique to study the queueing delay for sessions in case of a general session-length distribution. The analysis method is based on the combination of a generating-functions approach with the use of an infinite-dimensional state description. As a result, a closed-form expression for the mean session delay is obtained. The analysis is illustrated with a numerical example, based on real traces of file server traffic.
\end{abstract}

\section{INTRODUCTION}

All over the Internet, file servers provide for storage and access to files, both to human and computer users. When a certain file is requested, it can be segmented into smaller portions and then sent to the user that requested it. How much time is needed to completely send out the file depends on a variety of factors, such as: the server processor speed, the output link bandwidth, the number of parallel downloads, ... In order to optimize traffic or to guarantee a certain level of QoS, a profound understanding of the network and all its components is indispensable.

In this paper, we focus on the output buffer of a single file server link, modelled as a discrete-time infinite-capacity queueing system. Session-based arrivals are used to model the files being transferred. These sessions are groups of consecutive slots during which different packets from a single file are offered to the output buffer. A session therefore corresponds to a single file transfer.

The session-based arrival process was proposed in [1] and [2] to model the outgoing data buffer behavior of a web server, where a geometric distribution was assumed for the session lengths. It has been observed however that files on file and web servers are typically either small or very large and the file-size distribution is heavy-tailed, see e.g. [3], [4]. These phenomena cannot be modelled fully accurately by means of a geometric session-length distribution. The model we study in this paper therefore allows for general distributions of both the session length (expressed in slots) and the session bandwidth, i.e., the number of packets generated per slot during a session.
Such a general session-based arrival process does enable us to take into account the actual traffic characteristics, as observed from real traffic traces.

In a previous paper [5], we have studied the buffer content and the packet delay in a buffer with general session-based arrivals. The present paper elaborates on this previous work and derives analytical closed-form results for the session delay, i.e., the time between the arrival of the first packet of a session in the buffer and the completion of the transmission of the last packet of the session from the buffer.

Somewhat related analytical work on buffers with a train arrival process is found in [6], [7], [8] and [9], where messages arrive to the buffer at the rate of exactly one packet per slot. Also related is the correlated train arrival process considered in [10] and [11], where a finite number of users generate one packet per slot during active periods and no packets during passive periods. A simulation study on the file transmission duration in the web is reported in [12].

The paper is organized as follows. In Section II, we describe our mathematical model for a file server output buffer. In Section III, a summary of related previous results is given. Section IV concerns the analysis of the session delay. In Section V, the results are applied to study the session delay for an FTP server, based on traces of real FTP traffic. The paper is concluded in Section VI.

\section{Mathematical Model of OUtput BufFeR}

We model the output buffer of a file server link as a discretetime single-server queueing system with an infinite storage capacity for packets. Time is divided into fixed-length slots and transmissions from the output buffer can only start at slot boundaries.

General session-based arrivals in the output buffer are considered. Let $s_{k}$ be the number of newly started sessions during slot $k$. We assume the $s_{k}$ 's to be iid (independent and identically distributed), such that the distribution of $s_{k}$ is independent of $k$. The pgf (probability generating function) of the number of new sessions per slot is then given by

$$
S(z) \triangleq E\left[z^{s_{k}}\right]=\sum_{i=1}^{\infty} \operatorname{Prob}\left[s_{k}=i\right] z^{i} .
$$

The session lengths are also assumed to be iid random 
variables. We introduce

$$
\ell(i)=\operatorname{Prob}[\text { session length is } i \text { slots }], \quad L(z)=\sum_{i=1}^{\infty} \ell(i) z^{i} .
$$

The numbers of packets generated per slot during a session are assumed to be iid with pgf $P(z)$, where $P(0)=0$.

We define $a_{n, k}$ as the number of active sessions, that are active for exactly $n$ slots by the end of slot $k$. The following relationships then hold:

$$
a_{1, k}=s_{k}, \quad a_{n, k}=\sum_{i=1}^{a_{n-1, k-1}} c_{n-1, k}^{i}, \quad n>1,
$$

where $c_{n-1, k}^{i}$ denotes whether or not the $i$ th active session that was in its $(n-1)$ th slot during slot $k-1$, remains active during slot $k$. For convenience, we define $\pi(n-1)$ as the probability that a session that is already active for $n-1$ slots remains active for at least one more slot:

$$
\pi(n-1) \triangleq \frac{1-\sum_{i=1}^{n-1} \ell(i)}{1-\sum_{i=1}^{n-2} \ell(i)}
$$

The pgf of $c_{n-1, k}^{i}$ is then given by

$$
C_{n-1}(z)=1-\pi(n-1)+\pi(n-1) z, \quad n>1 .
$$

We also define $\tilde{a}_{k}$ as the total number of active sessions during slot $k$, such that $\tilde{a}_{k}=\sum_{n=1}^{\infty} a_{n, k}$.

The total number of packets generated during slot $k$, is defined as $m_{k}$ :

$$
m_{k}=\sum_{n=1}^{\infty} \sum_{i=1}^{a_{n, k}} p_{n, k}^{i}
$$

where $p_{n, k}^{i}$ denotes the number of packets generated during slot $k$ by the $i$ th session, active for exactly $n$ slots by the end of slot $k$.

The transmission time of a packet is geometrically distributed with parameter $1-\sigma$, such that the mean transmission time equals $1 / \sigma$. Due to the memoryless property of the geometric distribution an active transmission will end during the current slot with probability $\sigma$ and the transmission will continue with probability $1-\sigma$. We introduce the random variable $r_{k}$ as a Bernoulli variable with

$$
\operatorname{Prob}\left[r_{k}=0\right]=1-\sigma, \quad \operatorname{Prob}\left[r_{k}=1\right]=\sigma .
$$

Finally we introduce $u_{k}$ as the system content after slot $k$, i.e. at the beginning of slot $k+1$. The evolution of the system content is reflected in the equation

$$
u_{k}=m_{k}+\left(u_{k-1}-r_{k}\right)^{+} .
$$

From the equations (1)-(6), it can be concluded that the set of vectors $\left\{\left(a_{1, k}, a_{2, k}, \ldots, u_{k}\right)\right\}$ constitutes a Markov chain. Therefore, the system state after slot $k$ can be described simply by the infinite-dimensional vector $\left(a_{1, k}, a_{2, k}, \ldots, u_{k}\right)$.

\section{SUMMARY OF PREVIOUS WORK}

For the considered buffer system, the arrival process, the buffer content and the packet delay were studied in [5]. Here, we briefly summarize some results of [5] which are necessary for our present analysis.

First, the steady-state joint pgf $Q\left(x_{1}, x_{2}, \ldots, z\right)$ of the numbers of active sessions during a slot and the system content after the slot is given by

$$
\begin{aligned}
& Q\left(x_{1}, x_{2}, \ldots, z\right)=\frac{S\left(x_{1} P(z)\right)}{z}\left\{\sigma(z-1) p_{0}\right. \\
& \left.\quad+\Phi(z) Q\left(C_{1}\left(x_{2} P(z)\right), C_{2}\left(x_{3} P(z)\right), \ldots, z\right)\right\}
\end{aligned}
$$

where $\Phi(z) \triangleq \sigma+(1-\sigma) z$ and $p_{0}$ is the steady-state probability of an empty system, which can be determined as

$$
p_{0}=1-\frac{S^{\prime}(1) L^{\prime}(1) P^{\prime}(1)}{\sigma} \text {. }
$$

From (7), the mean system content is obtained as

$$
\begin{aligned}
& E[u]=-\frac{1}{2} S^{\prime}(1) P^{\prime}(1)\left[\sigma_{L}^{2}-L^{\prime}(1)+L^{\prime}(1)^{2}\right] \\
& +\frac{1}{2 \sigma(1-\rho)}\left\{\rho \sigma(2-\rho \sigma)+\left(\sigma_{P}^{2}-P^{\prime}(1)\right) S^{\prime}(1) L^{\prime}(1)\right. \\
& \left.\quad+\left(\sigma_{S}^{2} L^{\prime}(1)^{2} P^{\prime}(1)^{2}+\sigma_{L}^{2} S^{\prime}(1) P^{\prime}(1)^{2}\right)\right\},
\end{aligned}
$$

where $\sigma_{L}^{2}, \sigma_{S}^{2}$ and $\sigma_{P}^{2}$ are the variances of the session length, the number of new sessions and the session bandwidth respectively. The mean number of sessions that are active for exactly $n$ slots follows from (7) as

$$
E\left[a_{n}\right]=S^{\prime}(1)\left(1-\sum_{i=1}^{n-1} \ell(i)\right)=S^{\prime}(1) \Lambda(n),
$$

where we introduced $\Lambda(n) \triangleq \sum_{j=n}^{\infty} \ell(j)$ for convenience. Note also that $\sum_{n=1}^{\infty} \Lambda(n)=L^{\prime}(1)$.

\section{Analysis of the Mean Session Delay}

The session delay $d_{s}$ is the number of slots between the end of the arrival slot of the session's first packet and the end of the last transmission slot of the session's final packet. The pgf of the session delay is very tedious to determine and therefore we confine ourselves to the mean value. The mean session delay is then given by

$$
E\left[d_{s}\right]=\sum_{N=1}^{\infty} E\left[d_{s \mid N}\right] \ell(N),
$$

where $d_{s \mid N}$ denotes the session delay in the case the session takes $N$ slots. We distinguish two cases: $d_{s \mid 1}$ and $d_{s \mid N}$ for $N>1$. In what follows, we will refer to the tagged session as $T$. The arrival slot of session $T$ will be labeled $I$.

\section{A. Delay of a session of length 1}

In an FCFS queue, the session delay of a 1-slot session is given by the time, in slots, needed to transmit all packets in the buffer after the session's arrival slot, except for the ones that have arrived after the session's final packet. We define $q_{I}$ as the total number of packets that have arrived in slot $I$, but after the session's final packet. We define $u_{I}$ as the total 
system content after slot $I$. The mean session delay can then be expressed as:

$$
E\left[d_{s \mid 1}\right]=\frac{\left(E\left[u_{I}\right]-E\left[q_{I}\right]\right)}{\sigma} .
$$

Similarly, we introduce $a_{n, I}$ as the number of active sessions that have been active for exactly $n$ slots by the end of slot $I$. The joint pgf $G\left(x_{1}, x_{2}, \ldots, z\right)$ of the random variables $a_{n, I}$ and $u_{I}$ can then be found as

$$
\begin{aligned}
G\left(x_{1}, x_{2}, \ldots, z\right) & \triangleq E\left[\left(\prod_{n=1}^{\infty} x_{n}^{a_{n, I}}\right) z^{u_{I}}\right] \\
& =\frac{x_{1}}{S^{\prime}(1)} \frac{\partial}{\partial x_{1}} Q\left(x_{1}, x_{2}, \ldots, z\right) \\
& =\frac{x_{1} P(z) S^{\prime}\left(x_{1} P(z)\right)}{S^{\prime}(1) S\left(x_{1} P(z)\right)} Q\left(x_{1}, x_{2}, \ldots, z\right) .
\end{aligned}
$$

From this and the moment generating property of pgfs, the mean system content at the end of slot $I$ and the mean number of active sessions during slot $I$ are derived as

$$
\begin{aligned}
E\left[u_{I}\right] & =\frac{\partial}{\partial z} G(1,1, \ldots, 1)=E[u]+(1+R) P^{\prime}(1) ; \\
E\left[\tilde{a}_{I}\right] & =\sum_{n=1}^{\infty} \frac{\partial}{\partial x_{n}} G(1,1, \ldots, 1) \\
& =1+R+S^{\prime}(1) L^{\prime}(1)
\end{aligned}
$$

where we introduced the shorthand

$$
R \triangleq \frac{S^{\prime \prime}(1)-S^{\prime}(1)^{2}}{S^{\prime}(1)} .
$$

In order to calculate $E\left[d_{s \mid 1}\right]$, we still need to determine $E\left[q_{I}\right]$. Let $m_{I}$ be the total number of packets that arrive during slot $I$ and $m_{T}$ the number of packets in the tagged session $T$. Note that $q_{I}$ only depends on $m_{I}$ and $m_{T}$, due to the random order of packet arrivals in a certain slot. Specifically, we find that for $0 \leq \ell \leq m-m^{*}$,

$$
\operatorname{Prob}\left[q_{I}=\ell \mid m_{I}=m, m_{T}=m^{*}\right]=\frac{\left(\begin{array}{c}
m-\ell-1 \\
m^{*}-1
\end{array}\right)}{\left(\begin{array}{c}
m \\
m^{*}
\end{array}\right)} .
$$

If $p\left(j, l, m, m^{*}\right) \triangleq \operatorname{Prob}\left[\tilde{a}_{I}=j, q_{I}=\ell, m_{I}=m, m_{T}=m^{*}\right]$ and $q\left(j, m, m^{*}\right) \triangleq \operatorname{Prob}\left[\tilde{a}_{I}=j, m_{I}=m, m_{T}=m^{*}\right]$, we can then calculate $E\left[q_{I}\right]$ as

$$
\begin{aligned}
E\left[q_{I}\right] & =\sum_{j=1}^{\infty} \sum_{m=j}^{\infty} \sum_{m^{*}=1}^{m-j+1} \sum_{\ell=0}^{m-m^{*}} \ell p\left(j, \ell, m, m^{*}\right) \\
& =\sum_{j=1}^{\infty} \sum_{m=j}^{\infty} \sum_{m^{*}=1}^{m-j+1} \frac{m-m^{*}}{m^{*}+1} q\left(j, m, m^{*}\right) \\
& =\sum_{j=1}^{\infty} E\left[\frac{m_{I}-m_{T}}{m_{T}+1} \tilde{a}_{I}=j\right] \operatorname{Prob}\left[\tilde{a}_{I}=j\right],
\end{aligned}
$$

where we made use of the property

$$
\sum_{t=k}^{n}\left(\begin{array}{l}
t \\
k
\end{array}\right)=\left(\begin{array}{l}
n+1 \\
k+1
\end{array}\right)
$$

In order to compute the conditional mean in (16), we now introduce $\Omega_{j}(x, y)$ as the joint pgf of the random variables $m_{T}$ and $m_{I}$ conditioned on $\tilde{a}_{I}=j$ :

$$
\begin{aligned}
\Omega_{j}(x, y) & \triangleq E\left[x^{m_{T}} y^{m_{I}} \mid \tilde{a}_{I}=j\right] \\
& =E\left[x^{m_{T}} y^{m_{T}+\sum_{i=1}^{j-1} p_{I}^{i}} \mid \tilde{a}_{I}=j\right] \\
& =P(x y) P(y)^{j-1} .
\end{aligned}
$$

Note that the number of packets $p_{I}^{i}$ generated by a session $i$ during slot $I$, is independent of the total number of active sessions and the number of packets generated by other sessions during slot $I$. By means of $\Omega_{j}(x, y)$, we then find

$$
\begin{aligned}
E[ & {\left[\frac{m_{I}-m_{T}}{m_{T}+1} \mid \tilde{a}_{I}=j\right] } \\
& =\left.\left(\frac{\partial}{\partial y} \int_{0}^{1} \Omega_{j}(x, y) \mathrm{d} x\right)\right|_{y=1}-1+\int_{0}^{1} \Omega_{j}(x, 1) \mathrm{d} x \\
& =(j-1) P^{\prime}(1) \int_{0}^{1} P(x) \mathrm{d} x .
\end{aligned}
$$

We can now substitute this into (16) to obtain $E\left[q_{I}\right]$ as

$$
E\left[q_{I}\right]=P^{\prime}(1)\left(E\left[\tilde{a}_{I}\right]-1\right) \int_{0}^{1} P(x) \mathrm{d} x .
$$

Combination of the previous expressions finally leads to

$$
\begin{gathered}
E\left[d_{s \mid 1}\right]=\frac{P^{\prime}(1)}{\sigma}\left\{\frac{E[u]}{P^{\prime}(1)}+1+R\right. \\
\left.-\left(S^{\prime}(1) L^{\prime}(1)+R\right) \int_{0}^{1} P(x) \mathrm{d} x\right\} .
\end{gathered}
$$

\section{B. Delay of a session of length larger than 1}

The session delay of a session that lasts $N$ slots $(N>1)$, can be found as the total remaining transmission time needed to send all packets in the buffer at the end of the session's first packet's arrival slot, all packets arriving in the subsequent $N-2$ slots, and the packets arriving during the slot in which the session's final packet arrives, except for those arriving later than the mentioned packet. We define $u_{I}$ as the system content after slot $I$. Also we define $m_{I+i}$ as the total number of packet arrivals in the $(i+1)$ th slot of the session $(0 \leq i \leq N-1)$. Finally, we tag the slot in which the session's final packet arrives as slot $J$ and define $\bar{q}_{J}$ as the number of packets that arrive during slot $J$, but no later than the session's final packet. The mean session delay of a session of length $N$ can then be found as

$$
E\left[d_{s \mid N}\right]=\frac{\left(E\left[u_{I}\right]+\sum_{i=1}^{N-2} E\left[m_{I+i}\right]+E\left[\bar{q}_{J}\right]\right)}{\sigma} .
$$

Note that $E\left[u_{I}\right]$ is equal to the corresponding value determined in the previous section.

The terms $E\left[m_{I+i}\right]$ are equal to $P^{\prime}(1) \sum_{n=1}^{\infty} E\left[a_{n, I+i}\right]$, with

$$
a_{1, I+i}=s_{I+i}
$$




$$
\begin{aligned}
a_{i+1, I+i} & =\sum_{j=1}^{a_{i, I+i-1}-1} c_{i, I+i}^{j}+1, \quad 1 \leq i \leq N-1 ; \\
a_{n, I+i} & =\sum_{j=1}^{a_{n-1, I+i-1}} c_{n-1, I+i}^{j}, \quad n>1, n \neq i+1,
\end{aligned}
$$

where $s_{I+i}$ is defined as the number of new sessions during the $(i+1)$ th slot of session $T$. Note that the term 1 in expression (22) represents session $T$, which is known to continue. Taking mean values, we find

$$
\begin{aligned}
E\left[a_{1, I+i}\right] & =S^{\prime}(1) ; \\
E\left[a_{i+1, I+i}\right] & =1+\pi(i)\left(E\left[a_{i, I+i-1}\right]-1\right), \quad 1 \leq i \leq N-1 ; \\
E\left[a_{n, I+i}\right] & =\pi(n-1) E\left[a_{n-1, I+i-1}\right], \quad n>1, n \neq i+1 .
\end{aligned}
$$

Recursive application of the above equations then leads to

$$
\begin{aligned}
E\left[a_{i+1, I+i}\right] & =1+\frac{S^{\prime \prime}(1)}{S^{\prime}(1)} \Lambda(i+1), & & 1 \leq i \leq N-1 ; \\
E\left[a_{n, I+i}\right] & =S^{\prime}(1) \Lambda(n), & & n>1, n \neq i+1 .
\end{aligned}
$$

These expressions finally allow us to find $E\left[m_{I+i}\right]$ as

$$
\begin{aligned}
E\left[m_{I+i}\right]=P^{\prime}(1)\left\{1+S^{\prime}(1) L^{\prime}(1)+R \Lambda(i+1)\right\} & \\
& 1 \leq i \leq N-1 .
\end{aligned}
$$

Finally, we still need $E\left[\bar{q}_{J}\right]$. First, we define $m_{T}$ as the number of packets generated by the tagged session $T$ in its last slot $J$. Due to the random order of the packet arrivals within a certain slot, the number of packets arriving in $J$, but no later than the session's final packet, only depends on $m_{T}$ and the total number of packet arrivals during the last slot, $m_{J}$. Specifically, we find that for $m^{*} \leq \ell \leq m$,

$$
\operatorname{Prob}\left[\bar{q}_{J}=\ell \mid m_{J}=m, m_{T}=m^{*}\right]=\frac{\left(\begin{array}{c}
\ell-1 \\
m^{*}-1
\end{array}\right)}{\left(\begin{array}{c}
m \\
m^{*}
\end{array}\right)} .
$$

The determination of $E\left[\bar{q}_{J}\right]$ is quite similar to the determination of $E\left[q_{I}\right]$ in the previous section. Following similar steps, it can be shown that

$$
E\left[\bar{q}_{J}\right]=\sum_{j=1}^{\infty} E\left[\frac{m_{T}\left(m_{J}+1\right)}{m_{T}+1} \mid \tilde{a}_{J}=j\right] \operatorname{Prob}\left[\tilde{a}_{J}=j\right],
$$

where $\tilde{a}_{J}$ is the total number of active sessions during slot $J$. Since $m_{J}$ is identically distributed as $m_{I}$ from the previous section, former results can be reused to find

$$
\begin{aligned}
& E\left[\frac{m_{T}\left(m_{J}+1\right)}{m_{T}+1} \mid \tilde{a}_{J}=j\right] \\
& =j P^{\prime}(1)-(j-1) P^{\prime}(1) \int_{0}^{1} P(x) \mathrm{d} x,
\end{aligned}
$$

such that

$$
E\left[\bar{q}_{J}\right]=P^{\prime}(1) E\left[\tilde{a}_{J}\right]-P^{\prime}(1)\left(E\left[\tilde{a}_{J}\right]-1\right) \int_{0}^{1} P(x) \mathrm{d} x .
$$

Combining the above results and taking into account that $P^{\prime}(1) E\left[\tilde{a}_{J}\right]=E\left[m_{I+N-1}\right]$, we finally get $E\left[d_{s \mid N}\right]$ for $N>1$ as

$$
\begin{aligned}
& E\left[d_{s \mid N}\right]=\frac{P^{\prime}(1)}{\sigma}\left\{\frac{E[u]}{P^{\prime}(1)}+1+(N-1)\left(1+S^{\prime}(1) L^{\prime}(1)\right)\right. \\
& \left.-\left(S^{\prime}(1) L^{\prime}(1)+R \Lambda(N)\right) \int_{0}^{1} P(x) \mathrm{d} x+R \sum_{i=1}^{N} \Lambda(i)\right\} .
\end{aligned}
$$

\section{Mean session delay}

From (11), (19) and (26), the following explicit expression for the mean session delay can be derived:

$$
\begin{aligned}
& E\left[d_{s}\right]=\frac{P^{\prime}(1)}{\sigma}\left\{\frac{E[u]}{P^{\prime}(1)}+1+\left(L^{\prime}(1)-1\right)\left(1+S^{\prime}(1) L^{\prime}(1)\right)\right. \\
& -\left(S^{\prime}(1) L^{\prime}(1)+R \sum_{n=1}^{\infty} l(n) \Lambda(n)\right) \int_{0}^{1} P(x) \mathrm{d} x \\
& \left.\quad+R \sum_{n=1}^{\infty} \Lambda(n)^{2}\right\} .
\end{aligned}
$$

The double summations $(\Lambda(n)$ corresponds to a summation of its own) might look discouraging, but can be programmed in one single loop without nested loops.

\section{Case Study: Session Delay in an FTP Server}

The above model can be applied to predict the delay of a file download from a server with many simultaneous users. Consider an FTP server with a maximal transfer rate of 1 Gbit/s. Typically, such a server is connected to the Internet via a link with lower bandwidth (say $100 \mathrm{Mbit} / \mathrm{s}$ ), so a buffer is required at the output of the server. If a user requests a file from the server, it is first transferred to the buffer and from there transmitted over the link. As the transfer rate is limited, it is clear that the FTP traffic to the buffer forms a sessionbased arrival stream as in our model. To represent a realistic scenario, the parameters can be chosen as follows. We assume the packets have a fixed length of 100 bytes, which means the slot length is $(800 \mathrm{bit}) /(100 \mathrm{Mbit} / \mathrm{s})=8 \mu \mathrm{s}$. For the session bandwidth distribution, we take into account the ratio between the maximal input and output rate of the buffer: at most 10 packets can enter the buffer during a slot. We choose the ad hoc distribution

$P(z)=\frac{1}{20}\left(z+z^{2}+z^{3}\right)+\frac{3}{20}\left(z^{4}+z^{5}+z^{6}\right)+\frac{2}{20}\left(z^{7}+z^{8}+z^{9}+z^{10}\right)$,

with mean $P^{\prime}(1)=5.95$ packets. As a distinctive feature of our model we have that the session length distribution $L(z)$ is general as well. Let $F(z)$ be the distribution of the total number of packets generated during a session, then clearly

$$
F(z)=L(P(z)) .
$$

In our application, $F(z)$ is the distribution of the size of the requested files in 100-byte packets. We retrieve this distribution from a trace of measured FTP traffic, see [13], containing the incoming anonymous FTP connections (i.e. to port 21) to public file servers at the Lawrence Berkeley 


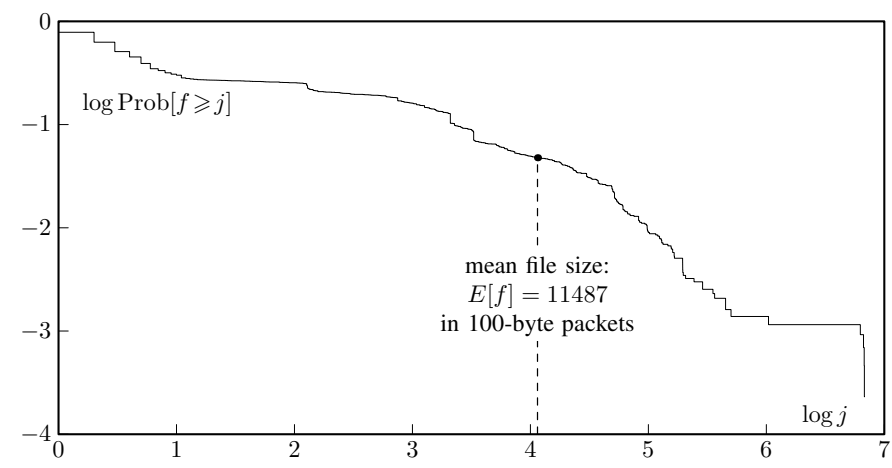

Fig. 1. Survival function $\operatorname{Prob}[f \geqslant j]$ of the FTP file size $f$ (in full 100byte packets) in the trace lbnl.anon-ftp.03-01-10 at [13], shown on a $\log -\log$ scale.

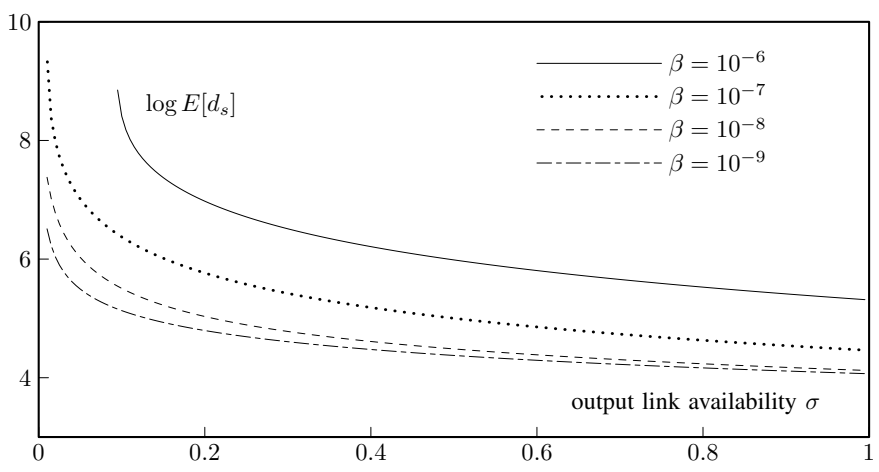

Fig. 2. Mean session delay $E\left[d_{s}\right]$ (in ms) versus the output link availability $\sigma$ on a $\log$ scale, for $\beta=10^{-9}, 10^{-8}, 10^{-7}, 10^{-6}$.

National Laboratory during a ten-day period in January 2003. From the trace of the first day, we extract the byte sizes of all transferred files and put them in a histogram with a bin size of 1 packet (i.e. 100 bytes). The mass function $\operatorname{Prob}[f=j]$ of the file size $f$ is taken as the probability of the $j$ th bin, resulting in the distribution shown in Fig. 1. Now, given $F(z)$ and $P(z)$, it remains to find $L(z)$ from (28). In general, no exact solution can be found so we choose a fit as follows:

$$
\ell(n)=\sum_{j=\left\lfloor(n-1) P^{\prime}(1)\right\rfloor+1}^{\left\lfloor n P^{\prime}(1)\right\rfloor} f(j),
$$

which ensures that $L^{\prime}(1) P^{\prime}(1) \approx F^{\prime}(1)$ with $L^{\prime}(1)=1931$ slots. Finally, we also assume that a new session starts each slot with probability $\beta$, i.e. $S(z)=1-\beta+\beta z$. The load of the buffer is then given by $\rho=\beta L^{\prime}(1) P^{\prime}(1) / \sigma=11490 \frac{\beta}{\sigma}$, where $\sigma$ is the output link availability.

In Fig. 2, the mean delay $E\left[d_{s}\right]$ experienced by a file in the output buffer is shown as a function of $\sigma$ and for different values of $\beta$. The curves have a vertical asymptote at $\sigma=$ $\beta L^{\prime}(1) P^{\prime}(1)$ where $\rho$ becomes equal to 1 . Higher values of $\beta$ correspond to higher system loads and therefore result in higher mean session delays. The parameter $\sigma$ has an opposite effect, since the load decreases with higher output channel availability. Note that in case of extremely small load (e.g. $\rho=0.115 \cdot 10^{-3}$ for $\beta=10^{-9}$ and $\sigma=1$ ) there are almost no concurrent file transfers, so $E\left[d_{s}\right] \approx L^{\prime}(1) P^{\prime}(1)=10^{4.06}$.

\section{Conclusions}

In this paper, we have developed an approximation technique to determine the mean file transmission delay in file server output buffers with general file sizes. We modelled the output buffer as a discrete-time infinite-capacity queueing system where the files were modelled as sessions. This allowed for an analytical technique, based on a generating-functions approach and an infinite-dimensional state description, to calculate the mean session delay.

\section{REFERENCES}

[1] L. Hoflack, S. De Vuyst, S. Wittevrongel and H. Bruneel, Analytic Traffic Model of Web Server, Electronics Letters, vol. 44, no. 1 (2008), pp. 61-62.

[2] L. Hoflack, S. De Vuyst, S. Wittevrongel and H. Bruneel, DiscreteTime Buffer Systems with Session-Based Arrival Streams, Performance Evaluation (2010), doi:10.1016/j.peva.2009.12.007.

[3] M. Arlitt and C. Williamson, Internet Web Servers: Workload Characterization and Performance Implications, IEEE-ACM Transactions on Networking, vol. 5, no. 5 (1997), pp. 631-645.

[4] T. Field, U. Harder and P. Harrison, Network Traffic Behaviour in Switched Ethernet Systems, Performance Evaluation, vol. 58, no. 23 (2004), pp. 243-260.

[5] S. Wittevrongel, S. De Vuyst and H. Bruneel, Analysis of DiscreteTime Buffers with General Session-Based Arrivals, Proc. of ASMTA 2009 (Madrid, 9-12 June 2009) Lecture Notes in Computer Science, vol. 5513 (2009), pp. 189-203.

[6] H. Bruneel, Calculation of Message Delays and Message WaitingTimes in Switching Elements with Slow Access Lines, IEEE Transactions on Communications, vol. 42, no. 2-4 (1994), pp. 255-259.

[7] S. Wittevrongel, Discrete-Time Buffers with Variable Length Train Arrivals, Electronics Letters, vol. 34, no. 18 (1998), pp. 1719-1721.

[8] B.D. Choi, D.I. Choi, Y. Lee and D.K. Sung, Priority Queueing System with Fixed-Length Packet-Train Arrivals, IEE ProceedingsCommunications, vol. 145, no. 5 (1998), pp. 331-336.

[9] J. Walraevens, S. Wittevrongel and H. Bruneel, A discrete-time Priority Queue with Train Arrivals, Stochastic Models, vol. 23, no. 3 (2007), pp. 489-512.

[10] F. Kamoun, Performance Analysis of a Discrete-Time Queuing System with a Correlated Train Arrival Process, Performance Evaluation, vol. 63, no. 4-5 (2006), pp. 315-340.

[11] F. Kamoun, Performance Evaluation of a Queuing System with Correlated Packet-Trains and Server Interruption, Telecommunication Systems, vol. 41, no. 4 (2009), pp. 267-277.

[12] R. Nossenson and H. Attiya, The Distribution of File Transmission Duration in the Web, International Journal of Communication Systems, vol. 17, no. 5 (2004), pp. 389-506.

[13] LBNL-FTP-PKT,

http://ee.lbl.gov/anonymized-traces.html 